\title{
An Actuator with Physically Variable Stiffness for Highly Dynamic Legged Locomotion
}

\author{
Jonathan W. Hurst, Joel E. Chestnutt and Alfred A. Rizzi \\ Carnegie Mellon University \\ Email: $\{$ jhurst, chestnutt, arizzi+\} $@$ ri.cmu.edu
}

\begin{abstract}
Running is a complex dynamical task which places strict design requirements on both the physical components and software control systems of a robot. This paper explores some of those requirements and illustrates how a variable compliance actuation system can satisfy them. We present the design, analysis, simulation, and benchtop experimental validation of such an actuator system. We demonstrate, through simulation, the application of our prototype actuator to the problem of biped running.
\end{abstract}

\section{INTRODUCTION}

A robot designed specifically for autonomous legged locomotion should be capable of highly dynamic running, jumping, and stumble recovery, and should achieve these capabilities while being energetically efficient. In order to accomplish these goals, we believe a leg spring of sufficient capacity to store the energy of a running gait is a necessity. Furthermore, we believe variable leg spring stiffness provides an important means for effective gait control.

One could concieve of two extremes of actuator design that would create these desirable properties: high-bandwidth actuators with all dynamics described by software control policies, or carefully designed mechanical systems with tuned natural dynamics that require no software control. The first method is flexible, although actuators with sufficiently high bandwidth and power capacity may not exist for the locomotion task. The second approach is quite inflexible, and requires extensive knowledge of the desired behavior before construction, but there is no bandwidth limit preventing the mechanism from behaving as intended at high frequencies. This method is taskspecific, and the resulting designs are unlikely to be able to perform the breadth of tasks required for running.

Our actuator design, shown in Figures 1 and 4, represents a carefully chosen balance between the two design extremes. The natural dynamics of the system are carefully designed and are utilized where possible, while the software controller adds energy that is lost in the mechanism, and creates behaviors that are not inherent in the natural dynamics. The actuator exhibits natural dynamics that are similar to those of animals, and is based on a previously developed mathematical model of running, shown in Figure 2, the spring loaded inverted pendulum (SLIP) [1], [2], [3].

\section{BACKGROUND}

Most research papers that analyse the mechanics of running base this analysis on some form of the SLIP model. This model describes the motion of the center of mass of a running animal [4], [5] or a running robot. The basic definition of running [6]

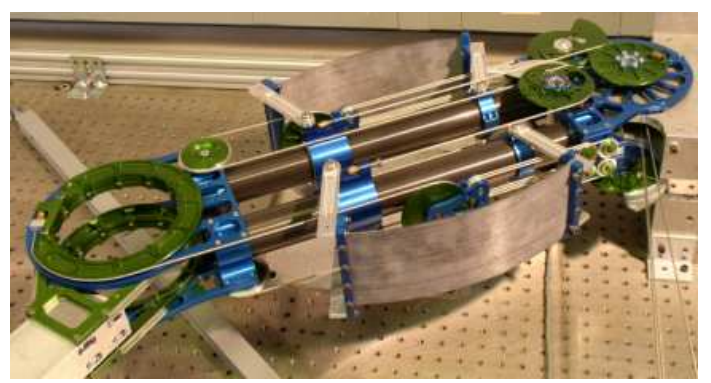

Fig. 1. The actuator prototype, mounted rigidly at the hip to an optical table

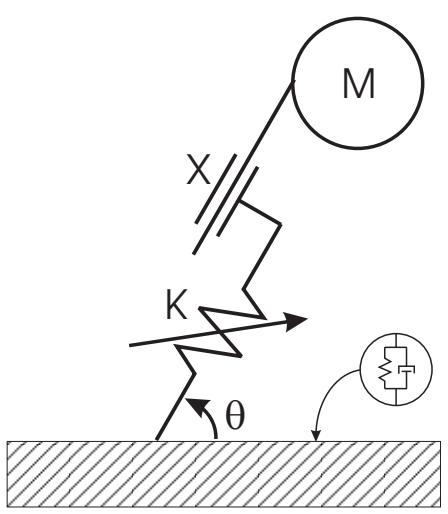

Fig. 2. The Spring Loaded Inverted Pendulum model of legged locomotion

is linked to the SLIP idea - energy is transferred from kinetic energy in the flight phase to spring energy in the stance phase, and vice versa. The natural physical instantiation of the SLIP model utilizes passive leg springs for this energy storage.

\section{A. Compliance and Running}

Physical series compliance is virtually necessary to achieve a successful running gait. Simulating compliance using a rigid actuator such as an electric gearmotor is not feasible for three reasons: bandwidth limitations, power output limitations, and energetic efficiency. The bandwidth limitation of an electric motor is due, in large part, to the high reflected inertia linked rigidly to the robot leg, making a correct dynamic response to impacts impossible. The power density of a physical spring is arbitrarily high, depending on its stiffness, making a compelling argument for combining the relatively high work capacity of a motor and power source with the high power density of a physical spring. Springs are particularly useful in rhythmic systems, because energy can be stored and released 
much more efficiently through a spring than if it were passed through the motor, transmission, and power electronics with each transfer. Animals store mechanical spring energy during a running gait, most likely for these reasons and more [7], [8], [9], [10].

\section{B. Physically Variable Compliance as a Method of Control}

While physical compliance is virtually a necessity for successful running, varying the compliance provides a useful tool for gait control. Animals have physically variable leg compliance (tuneable natural dynamics), and vary it to control running and hopping in certain situations [3], [4], [11], [12], [13], [14], [15], [16].

In a running gait, global vertical stiffness of the center of mass is the important factor [3]. It is influenced by changes in leg stiffness (caused in animals by muscle co-contraction and limb geometry) [12], touchdown angle, ground stiffness, and the number of legs on the ground at once. The global vertical stiffness is used by animals to control running speed, and increases with the square of the running speed [16]. Most research suggests that animals prefer to maintain leg stiffness over a range of running speeds [3], [13], [17], [18], using other methods to change global vertical stiffness; however, they do change leg stiffness when other methods are not available. For example, hopping or running on a surface of changing stiffness [4], [11], [13], hopping in place with varying frequency [4], or running at different speeds with constant stride length [17].

The running gait of a SLIP can be described by three parameters [1], and leg stiffness directly affects one or more of these parameters. For mathematical analysis in [1], leg length and angular velocity at bottom of stance along with the leg stiffness were chosen as the three parameters. Control of forward speed, stance duration, and flight duration was demonstrated experimentally in [19]. Another choice might be leg stiffness, hopping height, and stride length; there are many possible parameterizations that could be considered.

\section{The ACtuAtor Design}

The natural dynamics of a system are an inseparable part of its behavior, therefore the mechanical design is an essential part of the overall control system design. The actuator presented here was carefully designed as an integrated system of mechanism and software controller, with mechanical design choices made to match the mechanical model upon which the software controller is based. This model is illustrated in Figure 3, in two different forms: one rotational, one linear. The rotational model is physically similar to the prototype actuator, while the linear model is a simpler form that still captures the important properties of the system. In both models, the dynamics of the system controlling the pretension, $x_{3}$, are ignored and in the linear model, the spring stiffness, $K_{\text {eff }}$, is assumed to simply be a programmable value. Throughout the remainder of this paper we will use the notational conventions of Table 1 .

The actuator is essentially a single compliant joint, endowed with tuneable natural dynamics. There are two degrees of freedom, and two corresponding motors. One motor controls the spring pretension, corresponding to spring stiffness $\left(x_{3}\right.$ in Figure 3(a)), while the other motor controls the spring rest



(a) Rotational model

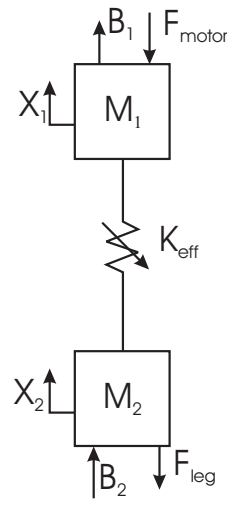

(b) Linear model
Fig. 3. Mechanical models of the physical actuator

\begin{tabular}{|r|l|}
\hline variable & description \\
\hline$\theta_{1}$ & Motor position \\
\hline$\theta_{2}$ & leg position \\
\hline$J_{1}$ & motor inertia \\
\hline$J_{2}$ & leg inertia \\
\hline$x_{3}$ & pretension \\
\hline$\Delta x$ & linear leg deflection, $r_{2} \theta_{2}-r_{1} \theta_{1}$ \\
\hline$z$ & $\begin{array}{l}\text { deflection of the cable after the pulley function: } \\
\left(x_{3}+\Delta x\right) \text { or }\left(x_{3}-\Delta x\right)\end{array}$ \\
\hline$F_{\text {eff }}\left(x_{3}, \Delta x\right)$ & knee force, $\tau_{e f f} / r_{2}$ \\
\hline$G(z)$ & spiral pulleys; spring position as a function of $z$ \\
\hline$y$ & deflection of the spring, before the pulleys \\
\hline$F_{y}(y)$ & force function of the spring \\
\hline$F_{z}(z)$ & force on the cable after the pulley function \\
\hline
\end{tabular}

TABLE I

position ( $\theta_{1}$ in Figure 3(a) and $x_{1}$ in Figure 3(b)). These two parameters, along with the leg angle at touchdown, are the parameters necessary to control SLIP model running.

Throughout the design process, minimizing weight has been a priority. The actuator is intended as a prototype leg for a bipedal robot with approximately 1 meter leg length and $30 \mathrm{~kg}$ total mass. Of this $30 \mathrm{~kg}, 20 \mathrm{~kg}$ are reserved for motors, batteries, and computing. This allotment leaves only $10 \mathrm{~kg}$ for the entire framework and mechanism, including springs and power transmission. To minimize weight, all joints contain thin-section bearings, which are very light for a given load rating. All parts are machined aluminum, with the main structural members (analogous to the femur) made of thinwall aluminum tube. The mass of this actuator prototype is approximately $4 \mathrm{~kg}$, and it is nearly $50 \%$ oversized.

\section{A. Mechanical Design}

Perhaps the most important aspect of the actuator is the physically variable series compliance. Energy is stored in fiberglass plates linked to spiral pulleys. The reduction ratio of the pulleys varies proportionally with the fiberglass spring deflection, to create a nonlinear spring function. Logarithmic spiral pulleys were chosen because the spring function of the bending fiberglass plates was unknown, because the desired spring function was unknown, and because two logarithmic spirals mesh correctly and provide a stiffening function [20]. These pulleys are modular components, and new pulleys can be designed to provide an arbitrary spring function. Given 
a general spring force function $F_{y}(y)$ and a general pulley transmission function $y=G(z)$, where $z$ is the extension of the cable out of the pulley, the force as a function of $z$ is

$$
F_{z}(z)=F_{y}(G(z)) \frac{\partial G}{\partial z}(z)
$$

allowing for suitable shaping of the overall effective spring function through choice of the pulley transmission function $G(z)$.

After creating a nonlinear spring function (for example, $F_{z}=K z^{2}$ ), placing two such spring/pulley systems in direct opposition results in a single effective torsional spring whose stiffness is determined by the pretension on each individual nonlinear spring. For the example quadratic spring, the resulting effective spring force is

$$
F_{\text {eff }}=4 K x_{3} \Delta x
$$

where $x_{3}$ represents the pretension on the two nonlinear springs and $\Delta x$ represents the deflection from their rest position. In this manner, the stiffness of the resulting system can be changed by adjusting the pretension. In practice, the rate at which this parameter can be varied depends on the actuator and transmission used. Our prototype is intended for relatively slow changes at low force, such as during the flight phase of a running gait.

Many of the mechanism design challenges are common ones; for example, minimizing friction, backlash, and inertia. In order to create a low-friction, zero-backlash system, our actuator utilizes a high-speed cable drive [21]. Any stretch in the cable effectively adds series compliance to the system, and is easily incorporated in the model. The cables may easily be routed around joints and may flex in two planes, allowing the motors to be located remotely. They are not constrained to a single degree of freedom, like standard belts or gears. Figure 4 shows the cable routing, illustrating the role of each motor in the tension of the two springs. Also shown is the fact that a displacement of the leg $\left(\theta_{2}\right.$ or $\left.x_{2}\right)$ results in either displacement of the motor $\left(\theta_{1}\right.$ or $\left.x_{1}\right)$ or displacement of the springs. There is a speed reduction between $\theta_{1}$ and $\theta_{2}$, not shown on the diagram; it is implemented using a combination of a block-and-tackle pulley mechanism and a difference in diameters between $\theta_{1}$ and $\theta_{2}$. The speed reduction is physically located near the knee joint, but diagrammatically located near the motor, $\theta_{1}$. All friction related to the speed reduction is applied to $\theta_{1}$ and corresponds to $B_{1}$, while the inertia corresponds to $J_{1}$. A speed reducer also amplifies the motor inertia by the square of the speed reduction; this amplification appears in the relatively large values of $M_{1}$. The transmission between $\theta_{2}$ and the springs has very low friction, and no speed reduction. Because the high-frequency behavior of the system is generally handled by the springs, low friction and inertia are most important in this part of the actuator. The low-frequency behaviors of the system are handled by the motor, and thus friction and inertia can be overcome by relatively low-bandwidth software compensation.

\section{B. Control System Design}

The control system is designed for the mechanical model shown in figure 3, and is intended to accomplish two basic

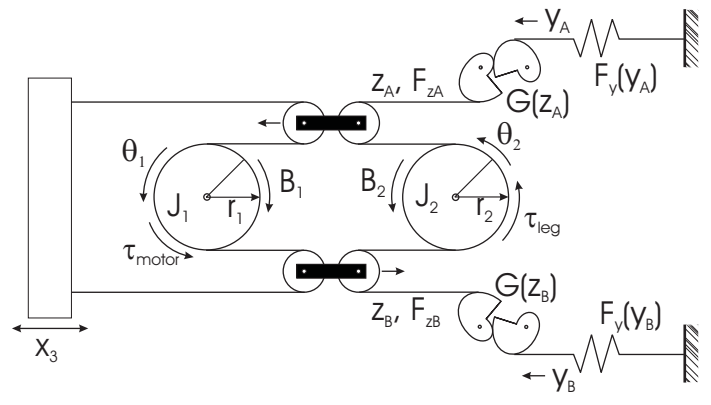

Fig. 4. Cable routing diagram of the actuator. $J_{1}$ and $J_{2}$ are pinned in place but can rotate freely; the spiral pulleys are also pinned in place but free to rotate. The remaining four pulleys are floating, and can move sideways as well as rotate. Refer to Table 1 for notation descriptions.

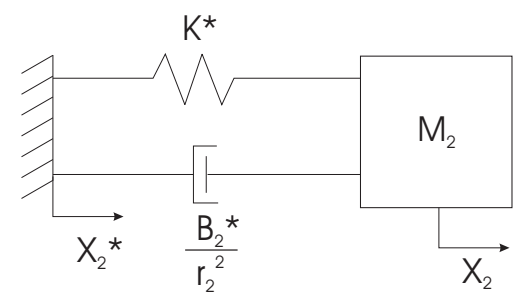

Fig. 5. The ideal case of our actuator, with three parameters: spring rest length, $x_{2}^{*}$, spring stiffness, $K^{*}$, and knee joint damping, $B^{*}$.

tasks. The first is to adjust the mechanism configuration so its physical properties match the commanded spring stiffness and rest length. The second is to actively control the motor $\left(x_{1}\right)$ to simulate the proper settings, when the mechanism is either out of adjustment range or in transition to the correct configuration. In the ideal setting, the motor will have to do very little work, and will allow the mechanical springs to store and release most of the energy in a running gait.

Adjusting the spring stiffness is accomplished with a simple PID position controller on the pretension motor, $x_{3}$. We ignore load forces and use a much larger motor than necessary for the current prototype. Because the specific position of this motor corresponds to a specific effective stiffness $K_{\text {eff }}$, no further control is currently required.

Adjusting spring rest length is accomplished using a simple PD controller on the position of $x_{1}$, with some added complexity when the pretension $x_{3}$ is not properly adjusted. In the ideal case, shown in Fig. 5, $x_{1}$ is fixed at the desired set point $x_{2}^{*}$, and the spring physically matches the desired spring stiffness $K^{*}$. There is some damping in the real system, and it is possible that some damping will be desired in the ideal system, so it has been added to the model, though it can easily be set to zero. To simulate this system, we must control $x_{1}$ so that it will simulate the desired spring stiffness if the physical system does not match our desired system. The torque on the leg applied by the physical system should match the torque on the leg applied by the desired system,

$$
K_{\text {eff }}\left(\Delta x, x_{3}\right) r_{2}+\frac{B_{2}}{r_{2}} \dot{x}_{2}=\left(x_{2}-x_{2}^{*}\right) K^{*} r_{2}+\frac{B_{2}^{*}}{r_{2}} \dot{x}_{2} .
$$

Assuming the dependence of $K_{\text {eff }}$ on $\Delta x$ is linear (recall that $\Delta x=x_{2}-x_{1}$ ), we can solve this equation for $x_{1}$ to calculate the desired position, $x_{1}^{*}$, 


$$
x_{1}^{*}=\frac{K^{*}}{K_{\text {eff }}}\left(x_{2}^{*}-x_{2}\right)-\frac{B_{2}^{*}-B_{2}}{K_{\text {eff }} r_{2}^{2}} \dot{x}_{2}+x_{2} .
$$

We then apply a PD controller on $x_{1}$ to move it to the desired position, along with a spring cancellation force to hold it against the force applied by the springs:

$$
F_{\text {com }}=K_{P}\left(x_{1}^{*}-x_{1}\right)+K_{D}\left(\dot{x}_{1}^{*}-\dot{x}_{1}\right)-K_{\text {eff }}\left(\Delta x, x_{3}\right) \text {. }
$$

With the spring cancellation force, the PD control can adjust $x_{1}$ as if it were an independent mass, without the attached spring and associated dynamics.

There are two limitations in this approach that introduce error. First, because $K_{\text {eff }}$ is a function composed of the logarithmic spiral pulleys and the unknown fiberglass spring function, it is necessarily an approximation. We used an approximation of $K_{\text {eff }}$ that is linear in $\Delta x$, so our match of $K_{\text {eff }}$ to $K^{*}$ will only be as good as our approximation fits the actual relationship defining $K_{\text {eff }}$. In addition, when simulating a spring stiffness that is outside the physical range of the actuator, the calculated location $x_{1}^{*}$ will only be correct to the accuracy of the approximation.

The second source of error comes from the bandwidth limitation on $x_{1}$. When trying to simulate a stiffness at high frequency, the inertia of $M_{1}$ will limit the acceleration of $x_{1}$, and the system will revert to the behavior of its natural dynamics, instead of the desired behavior. However, because the stiffness of the actuator is adjustable, this error can only happen when the desired stiffness is outside the range of the mechanism, or when the mechanism is in the process of adjusting to the correct stiffness.

\section{Simulation, Results AND COMPARison}

Because this actuator is currently bolted to an optical table, and is not part of a running robot that exhibits all of the desired dynamic properties, some experimental evidence is required to support the assertion that it does indeed match the SLIP model and would work effectively in a dynamic legged robot. We begin by measuring and testing certain aspects of the physical prototype, so these measurements may be used in a software simulation. The actuator is then compared to the simulation at a range of frequencies, showing that the software-electricalmechanical system behaves very similarly to the simulation we have developed. Further analysis is done using the simulation, by using tests that would be difficult to apply in the real world. Finally, the actuator simulation is tested in a simulation of a bipedal runner, providing evidence that the actuator could be effectively used on a physical running robot.

\section{A. Static Compliance Characterization}

We modeled the spring function of the actuator in order to create an accurate simulation. The function was measured using a load cell mounted against the leg of the actuator, recording applied force of the leg while cycling through a range of set points at different pretension settings. As can be seen in Figure 6 , the spring function $F_{\text {eff }}=K_{\text {eff }}(\Delta x)$ gets stiffer as the pretension increases. The slope is not quite linear, which violates our earlier assumption. This error can be remedied by manufacturing new spiral pulleys to create a new

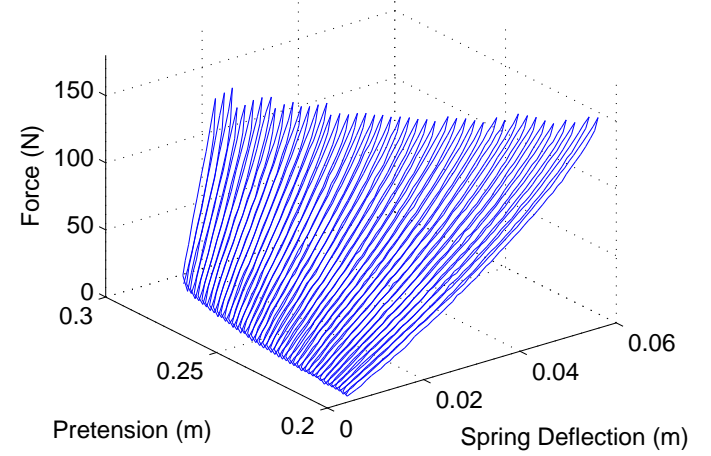

Fig. 6. Measured spring force as a function of deflection, $\Delta x$, and pretension, $x_{3}$

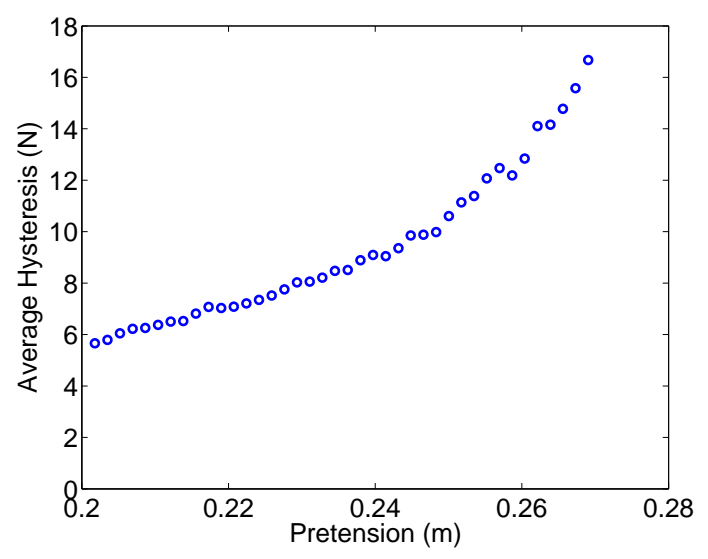

Fig. 7. Measured average hysteresis as a function of pretension, $x_{3}$

$G(z)$, resulting in the correct desired output function, $F_{z}(z)$. As in the earlier example, creating a quadratic spring function would result in an overall effective spring constant $K_{\text {eff }}$ which is linear and stiffens with pretension changes.

As is shown on the stiffness function graph in Figure 6, there is some hysteresis due to friction. The friction is caused by the sliding of the spring guides and the rolling of the bearings on the nonlinear pulleys, and could be depicted with an additional damper and stiction model in parallel with the springs in Figure 3. The level of hysteresis as a function of pretension is depicted in Figure 7. This figure was created by averaging the hysteresis at each of a range of pretension settings, because the hysteresis was relatively constant over a range of deflections at a given pretension value. As might be expected, hysteresis increases as the pretension (and forces applied to all bearings and cables) gets larger. For each pretension value, forces on the order of $180 \mathrm{~N}$ were applied-so at very high pretension, hysteresis was nearly $10 \%$ of the largest applied force. The knee friction $B_{2}$ is very small, so the spring hysteresis would account for most of the energy lost to friction in a running gait. In comparison to air cylinders used in some hopping robots [22], which can exhibit energy loss due to seal friction on the order of $25 \%$, this loss is relatively small. 

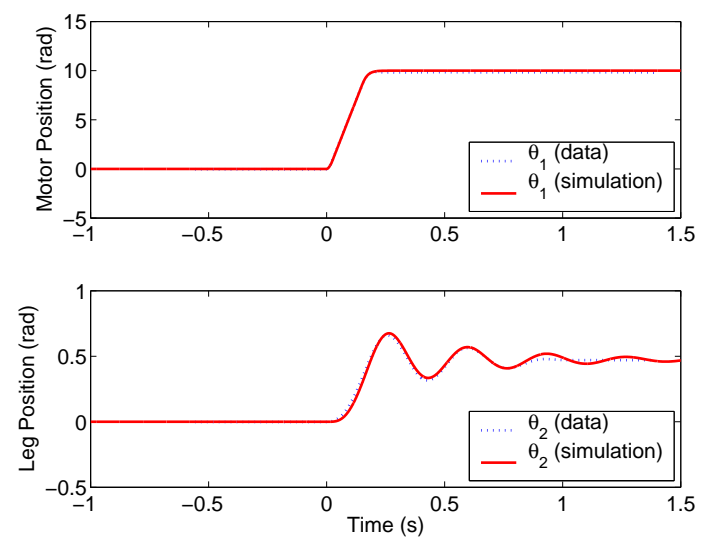

Fig. 8. Measured and simulated actuator position response to a step command in spring rest length

\section{B. Dynamic Actuator Simulation}

A dynamic simulation of the actuator prototype was created using the rotational model shown in Figure 3, along with the measured stiffness function and the values shown in Table 2. The values in Table 2 were calculated from the SolidWorks ${ }^{1}$ design of the actuator, the published properties of our motor, and from several system identification tests. After adding these values to the computer simulation, its response was tested with a step-input on the spring set point and compared to the same input on the physical actuator. Figure 8 shows the response of both $\theta_{1}$ and $\theta_{2}$.

\begin{tabular}{|r|c|c|l|}
\hline variable & value & variable & value \\
\hline \hline$J_{1}$ & $0.00134 \mathrm{~kg} \cdot \mathrm{m}^{2}$ & $M_{1}=\frac{J_{1}}{r_{1}^{2}}$ & $59.6 \mathrm{~kg}$ \\
\hline$J_{2}$ & $0.085 \mathrm{~kg} \cdot \mathrm{m}^{2}$ & $M_{2}=\frac{J_{2}}{r_{2}^{2}}$ & $8.5 \mathrm{~kg}$ \\
\hline$B_{1}$ & $0.0517 \mathrm{~N} \cdot \mathrm{m} \cdot \mathrm{s} / \mathrm{rad}$ & $r_{1}$ & $0.00474 \mathrm{~m}$ \\
\hline$B_{2}$ & $0.38 \mathrm{~N} \cdot \mathrm{m} \cdot \mathrm{s} / \mathrm{rad}$ & $r_{2}$ & $0.1 \mathrm{~m}$ \\
\hline
\end{tabular}

TABLE II

PHYSICAL PROPERTIES USED FOR SIMULATION

To illustrate performance over a range of frequencies, Bode plots of the actuator gain were created with motor position, $x_{1}$, as input and leg position, $x_{2}$, as output. The motor position, $x_{1}$, was commanded to track a sine wave beginning at a frequency of $1 \mathrm{~Hz}$ and cycling through to $6 \mathrm{~Hz}$, and the position of $x_{2}$ was recorded. Figure 9 shows the resulting Bode plots, for both the simulation and the actuator, at three different values of $K_{\text {eff }}$. Although this test does not provide great insight into the performance of the actuator, it does serve to compare it and the simulation and verify that both behave similarly at a range of frequencies. The basic structure of the graphs between the simulator and the actuator is very similar. The values do not match exactly, and we speculate that the most likely reason for this discrepancy is an inaccurate model of friction and stiction.

One major benefit of this actuator design is that aspects of its natural dynamics are programmable, and should be exhibited accurately to a high bandwidth. Figure 10 shows the ratio of expected to desired impedance of the actuator

\footnotetext{
${ }^{1}$ SolidWorks is a registered trademark of SolidWorks Corporation
}

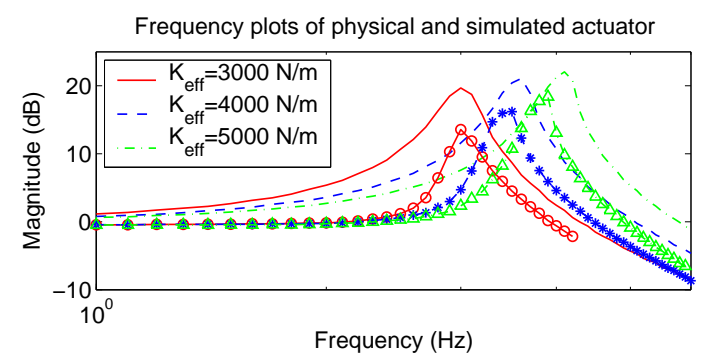

Fig. 9. Comparison of the position transfer function (ratio of motor position to leg position) for the physical and simulated actuators. Data points are depicted on experimental data from the physical actuator; Circles are 3000 $\mathrm{N} / \mathrm{m}$, stars are $4000 \mathrm{~N} / \mathrm{m}$, triangles are $5000 \mathrm{~N} / \mathrm{m}$.
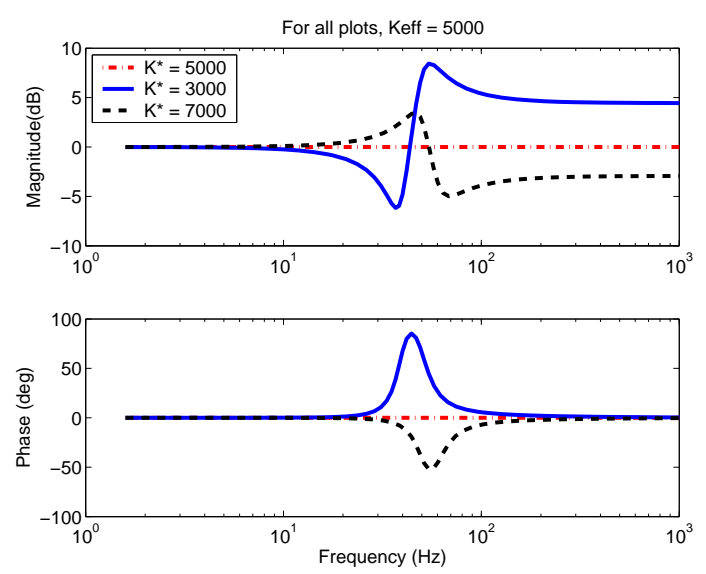

Fig. 10. Magnitude and phase of the ratio of actual to desired impedance as a function of frequency, with the commanded spring stiffness $K^{*}$ higher, lower, and the same as the physical system stiffness $K_{\text {eff }}$

simulation measured over a range of frequencies. The actuator stiffness $K_{\text {eff }}$ is a constant for the three plots, with different values for $K^{*}$; one set higher than $K_{\text {eff }}$, one lower, and one tuned to the same value. At low frequencies, the motor is capable of simulating the commanded spring constant $K^{*}$ by compensating actively for the discrepancy. As the frequency increases, it is apparent from the figure that the phase begins to lag or lead, while the exhibited stiffness begins to diverge from the commanded stiffness $K^{*}$. At very high frequencies, the motor does virtually nothing to affect the exhibited spring stiffness, which converges to the natural dynamics of the system, $K_{\text {eff }}$. For the tuned system with natural dynamics matching the commanded stiffness, the behavior does not change with frequency.

\section{Running Simulation}

After the similarity between actuator simulation and actuator prototype was established, we tested the simulation in a running application. A simulated runner was created using SD/FAST ${ }^{2}$, creating basic 2-link legs with a knee and hip joint attached to a body. An ideal spring was placed at each knee, with set point and stiffness as programmable variables that can change instantaneously. Running controllers were

\footnotetext{
${ }^{2} \mathrm{SD} / \mathrm{FAST}$ is a trademark of Symbolic Dynamics, Inc. and ParametricTechnology Corporation
} 


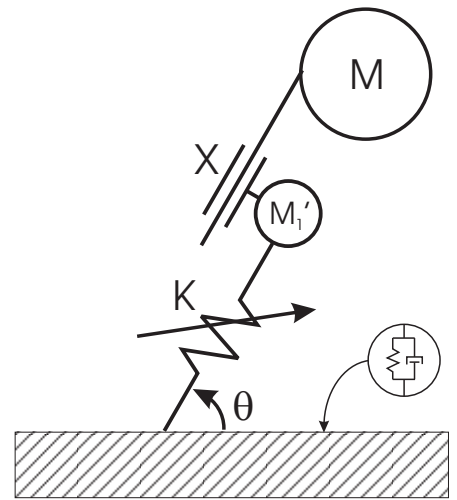

(a) Linear model

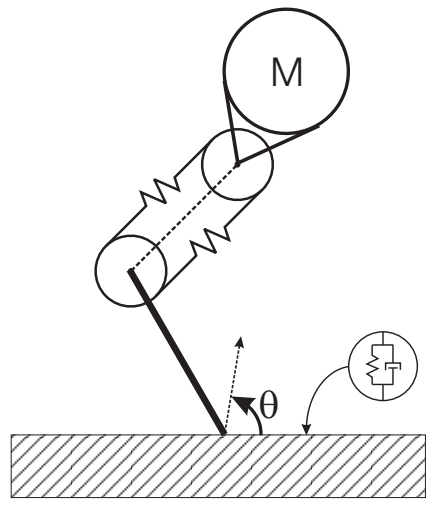

(b) Rotational model

Fig. 11. Equivalent SLIP models, both linear and rotational, incorporating actuator models as shown in Figure 3.

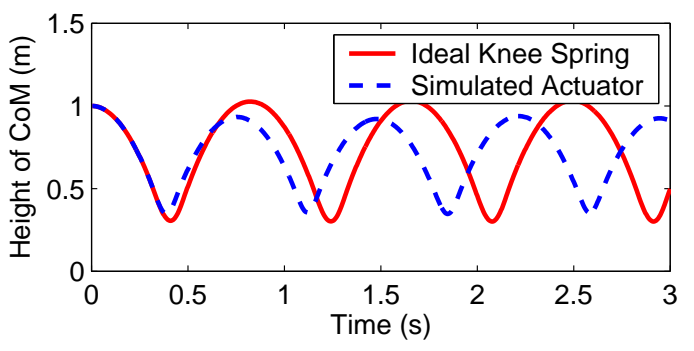

Fig. 12. Comparison of hopping behavior between ideal knee spring and realistic actuator model in hopping simulation

adapted from [22], and calculate the leg touchdown angle and energy insertion required to stabilize a running gait at a certain hopping height. Leg stiffness was held constant. After tuning the controller and adding user-inputs to change running speed and hopping height, the runner maintained a stable gait within reasonable bounds. We then replaced the ideal spring at each knee with the full dynamic simulation of the actuator, as shown in Figure 11(b). The primary difference between this model and the original SLIP shown in Figure 2 is the presence of the reflected motor inertia, depicted as $M_{1}^{\prime}$ in Figure 11(a). Figure 12 shows the vertical position of the center of mass over time. The simulated actuator has a slightly lower hopping height and resulting shorter stride length, caused by lower energy insertion with each hop. This is because, unlike an ideal spring, a discrete change to the commanded spring set point does not cause an instantaneous change the actual spring set point, due to the motor inertia $M_{1}^{\prime}$. However, this discrepancy is relatively minor, and easily accounted for in future controller revisions.

\section{Conclusions}

Physical compliance and mechanical energy storage are crucial for a successful running gait, while variable compliance is a useful control parameter for SLIP model running. The actuator design presented here has mechanical energy storage, tunable compliance, low friction, and zero backlash. Within its range of physical compliance, the actuator has virtually no bandwith limitations and is similar to an ideal SLIP. Based on the results presented, the concepts embodied in this actuator prototype could result in an effective actuation method for highly dynamic legged locomotion.

There are still many unanswered questions for further investigation. For example, we would like to determine what stiffness range is required for robust running on various surfaces, the necessary rate of stiffness adjustment, and the desired spring function.

\section{ACKNOWLEDGEMENTS}

This work is supported in part by an NSF Graduate Fellowship held by the first author and by the Robotics Institute of Carnegie Mellon University

\section{REFERENCES}

[1] W. J. Schwind and D. E. Koditschek, "Characterization of monopod equilibrium gaits," in Proceedings of the 1997 IEEE International Conference on Robotics and Automation, Albequerque, New Mexico, April 1997, pp. 1986-1992.

[2] R. Blickhan, "The spring-mass model for running and hopping," Journal of Biomechanics, vol. 22, no. 11/12, pp. 1217-1227, 1989.

[3] T. A. McMahon and G. C. Cheng, "The mechanics of running: How does stiffness couple with speed?" Journal of Biomechanics, vol. 23, pp. 65-78, 1990.

[4] R. J. Full and C. T. Farley, "Musculoskeletal dynamics in rhythmic systems - a comparative approach to legged locomotion," in Biomechanics and Neural Control of Posture and Movement, J. M. Winters and P. E. Crago, Eds. New York: Springer-Verlag, 2000.

[5] R. Blickhan and R. J. Full, "Similarity in multilegged locomotion: Bouncing like a monopode," Journal of Comparative Physiology, pp. 509-517, 1993.

[6] J. R. Hutchinson, D. Famini, R. Lair, and R. Kram, "Are fast-moving elephants really running?" Nature, no. 422, pp. 493-494, 2003.

[7] G. A. Cavagna, H. Thys, and A. Zamboni, "The sources of external work in level walking and running," Journal of Physiology, vol. 262, pp. 639-657, 1976.

[8] G. A. Cavagna, "Elastic bounce of the body," Journal of Applied Physiology, vol. 29, no. 3, pp. 279-282, 1970.

[9] G. A. Cavagna, N. C. Heglund, and C. R. Taylor, "Mechanical work in terrestrial locomotion: Two basic mechanisms for minimizing energy expenditure," American Journal Physiology, vol. 233, no. 5, pp. R243R261, 1977.

[10] T. A. McMahon, "Mechanics of locomotion," The International Journal of Robotics Research, vol. 3, no. 2, pp. 4-28, 1984.

[11] D. P. Ferris and C. T. Farley, "Interaction of leg stiffness and surface stiffness during human hopping," The American Physiological Society, pp. 15-22, 1997.

[12] C. T. Farley, H. H. P. Houdijk, C. V. Strien, and M. Louie, "Mechanism of leg stiffness adjustment for hopping on surfaces of different stiffnesses," The American Physiological Society, pp. 1044-1055, 1998.

[13] D. P. Ferris, M. Louie, and C. T. Farley, "Running in the real world: adjusting leg stiffness for different surfaces," in Proc. R. Soc. Lond., vol. 265, January 1998, pp. 989-993.

[14] T. A. McMahon and P. R. Greene, "The influence of track compliance on running," Journal of Biomechanics, vol. 12, pp. 893-904, 1979.

[15] T. A. McMahon, G. Valiant, and E. C. Frederick, "Groucho running," Journal of Applied Physiology, vol. 62, pp. 2326-2337, 1987.

[16] T. A. McMahon, "The role of compliance in mammalian running gaits," Journal of Experimental Biology, vol. 115, pp. 263-282, 1985.

[17] C. T. Farley and O. Gonzalez, "Leg stiffness and stride frequency in human running," Journal of Biomechanics, vol. 29, no. 2, pp. 181-186, 1995.

[18] C. T. Farley, J. Glasheen, and T. A. McMahon, "Running springs: Speed and animal size," Journal of Experimental Biology, vol. 185, pp. 71-87, 1993.

[19] J. K. Hodgins and M. H. Raibert, "Adjusting step length for rough terrain," in IEEE Transactions on Robotics and Automation, vol. 7, no. 3, 1991.

[20] N. P. Chironis, Mechanisms, Linkages, and Mechanical Controls. McGraw Hill, 1965, ch. Types of Noncircular Gears, pp. 241-244.

[21] W. T. Townsend and J. K. Salisbury, "Mechanical bandwidth as a guidline to high-performance manipulator design," in IEEE, 1989, pp. $1390-1395$.

[22] M. Raibert, Legged Robots That Balance. Cambridge, Mass.: MIT Press, 1986. 